\title{
Calcium-induced Translocation of Synaptic Vesicles to the Active Site
}

\author{
J. H. Koenig, Kageyuki Yamaoka, ${ }^{a}$ and Kazuo Ikeda \\ Division of Neurosciences, Beckman Research Institute of the City of Hope, Duarte, California 91010
}

\begin{abstract}
The effect of increasing $\left[\mathrm{Ca}^{2+}\right]_{\circ}$ on the positioning of synaptic vesicles relative to the active site in resting coxal neuromuscular junctions of Drosophila was investigated. In normal saline $\left(1.8 \mathrm{~mm} \mathrm{Ca}^{2+}\right)$ only a very small percentage of sites possess a vesicle docked under the dense body plate ciose to the plasma membrane in a readily releasable positlon. However, after exposure to elevated $\mathrm{Ca}^{2+}$ salines (3.6, $9,18 \mathrm{~mm}$ ), an increase in the number of active sites possessing docked vesicles was observed. Also, an increase in the average number of docked vesicles/site was seen. Intracellular recordings from coxal muscle fibers in normal saline and elevated $\mathrm{Ca}^{2+}$ salines were made, and it was observed that exposure to elevated $\mathrm{Ca}^{2+}$ saline caused an increase in miniature excitatory junction potential (mejp) frequency and in multiquantal and clustered mejps. Thus, when the number of active sites possessing docked vesicles increases, the frequency of spontaneous release also increases. Furthermore, when the number of docked vesicles/site increases, the number of multiquantal mejps increases. The data suggest that $\mathrm{Ca}^{2+}$ may be involved in vesicle translocation to the active site, and that the concentration of $\mathrm{Ca}^{2+}$ in the terminal may regulate the number of active sites that possess readily releasable vesicles. The effects of increasing the number of docked vesicles on spontaneous release characteristics are discussed.
\end{abstract}

[Key words: calcium, synapsin, active site, synaptic transmission, synaptic vesicle, readily releasable vesicles, vesicle translocation]

The most well-accepted explanation for transmitter release is the vesicle hypothesis (del Castillo and Katz, 1956), which proposes that transmitter is stored in synaptic vesicles, which fuse with the presynaptic membrane and release their contents by exocytosis upon depolarization of the presynaptic plasma membrane. An additional hypothesis associated with the release mechanism is the "Ca ${ }^{2+}$ hypothesis" (Katz and Miledi, 1965), which states that depolarization of the presynaptic nerve terminal opens $\mathrm{Ca}^{2+}$ channels, resulting in an influx of $\mathrm{Ca}^{2+}$ and

\footnotetext{
Received Sept. 4, 1992; reviseed Nov. 17, 1992; accepted Nov. 23, 1992.

We thank Ms. Grace Hong and Ms. Cecilia Macias for their excellent technical help and Ms. Sharyn Webb for her excellent secretarial help. This work was supported by U.S. Public Health Service NIH Grant NS 18856, NSF Grant BNS8415920 , and a Beckman Research Institute grant

Correspondence should be addressed to Jane Koenig, Ph.D., Division of Neurosciences, Beckman Research Institute of the City of Hope, 1450 East Duarte Road, Duarte, CA 91010.

- Present address: Natural Science Laboratory, Toyo University, Asaka, Saitama-ken, Japan.

Copyright (C 1993 Society for Neuroscience $0270-6474 / 93 / 132313-10 \$ 05.00 / 0$
}

a transient rise in the intracellular $\mathrm{Ca}^{2+}$ concentration adjacent to the plasma membrane. The $\mathrm{Ca}^{2+}$ binds to a receptor molecule and, by an unknown mechanism, triggers the exocytotic release of transmitter (for review, see Augustine et al., 1987).

Various processes of the release mechanism may involve $\mathrm{Ca}^{2+}$. One such process is the translocation of vesicles to the release site (Berl et al., 1973). The vesicle-associated protein, synapsin I, has received particular attention recently in this regard. It has been proposed that synapsin I may link synaptic vesicles to cytoskeletal elements, and by the phosphorylation-dependent dissociation of synapsin I from vesicles, $\mathrm{Ca}^{2+}$ could be involved in regulating vesicle availability (for review, see Atlas, 1990). While indirect evidence may suggest a role for $\mathrm{Ca}^{2+}$ in vesicle translocation to the release site from a more interiorly located position in the terminal, direct evidence actually demonstrating $\mathrm{Ca}^{2+}$-mediated vesicular movement has been difficult to obtain. In chromaffin cells (Edwards et al., 1984) and mast cells (Curran and Brodwick, 1984), no gross movement of secretory vesicles could be detected during exocytosis, and in neurons, the small size of the vesicles is prohibitive to direct observation of vesicular movement in vivo.

The present article presents evidence that $\mathrm{Ca}^{2+}$ is indeed involved in vesicle translocation to the active (release) site. The experiment takes advantage of the fact that in Drosophila coxal neuromuscular junctions, only a small percentage of the total active sites possesses a vesicle docked under the dense body in a readily releasable position. Thus, almost all of the vesicles are located more interiorly in the cytoplasm. In this article, it is shown that with exposure to high- $\mathrm{Ca}^{2+}$ saline, a dramatic increase occurs in the number of sites possessing docked vesicles, as well as the number of docked vesicles per site. Intracellular recordings reveal that, in this condition, the frequency of spontaneous release increases and also an increase in clustered and synchronized (multiquantal) spontaneous release occurs. Thus, $\mathrm{Ca}^{2+}$ appears to affect the number of vesicles that are in a readily releasable position at the active sites, and this, in turn, affects spontaneous release characteristics.

\section{Materials and Methods}

The experimental animals used in this work were 4-6-d-old, wild-type (Oregon-R) female Drosophila melanogaster. The sternal anterior rotator muscle of the first leg, one of six coxal muscles, was used for both the morphological and physiological experiments. The muscle lies under the prothoracic preepisternum in the ventral thorax. It attaches laterally to the anterior base of the coxa and medially to the median plate of the sternal apophysis. It is singly innervated by a motor neuron located in the prothoracic ganglion, which sends its axon through the first leg nerve to the coxal nerve. Each fiber is innervated by a single unbranching axon of this motor neuron, which runs parallelly along the fiber making hundreds of en passant-type synapses. 

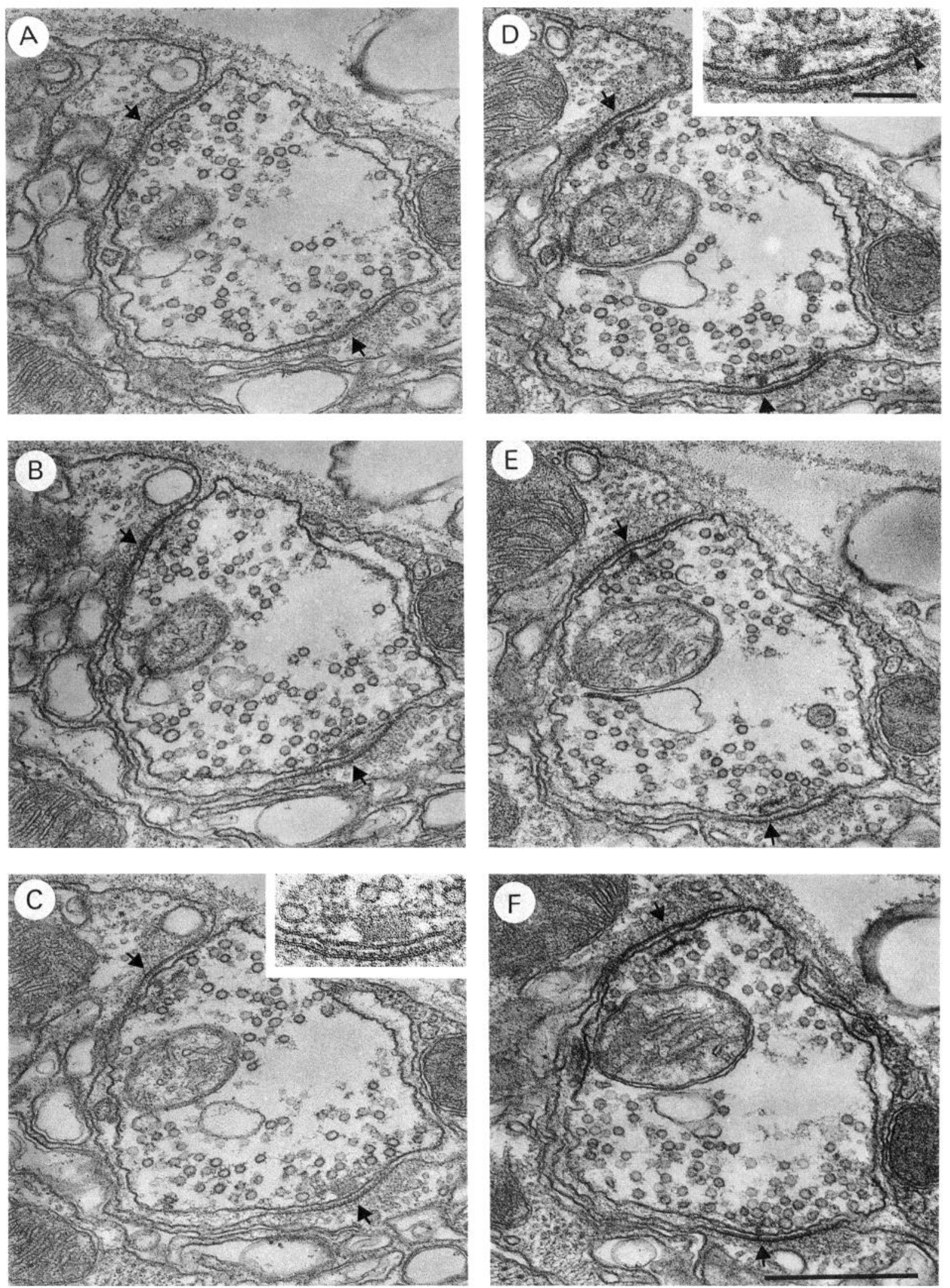

Figure 1. Serial sections through a typical resting coxal synapse (nerve cut). Note the two active sites with presynaptic dense bodies (arrows). Insets in $C$ and $D$ demonstrate the dense body at higher magnification. Arrowhead in inset $D$ points to brush-like attachment of dense body plate to the plasma membrane. Scale bars: inset $D, 100 \mathrm{~nm}(112,500 \times)$ for insets $C$ and $D ; F, 0.5 \mu \mathrm{m}(54,000 \times)$ for $A-F$. 

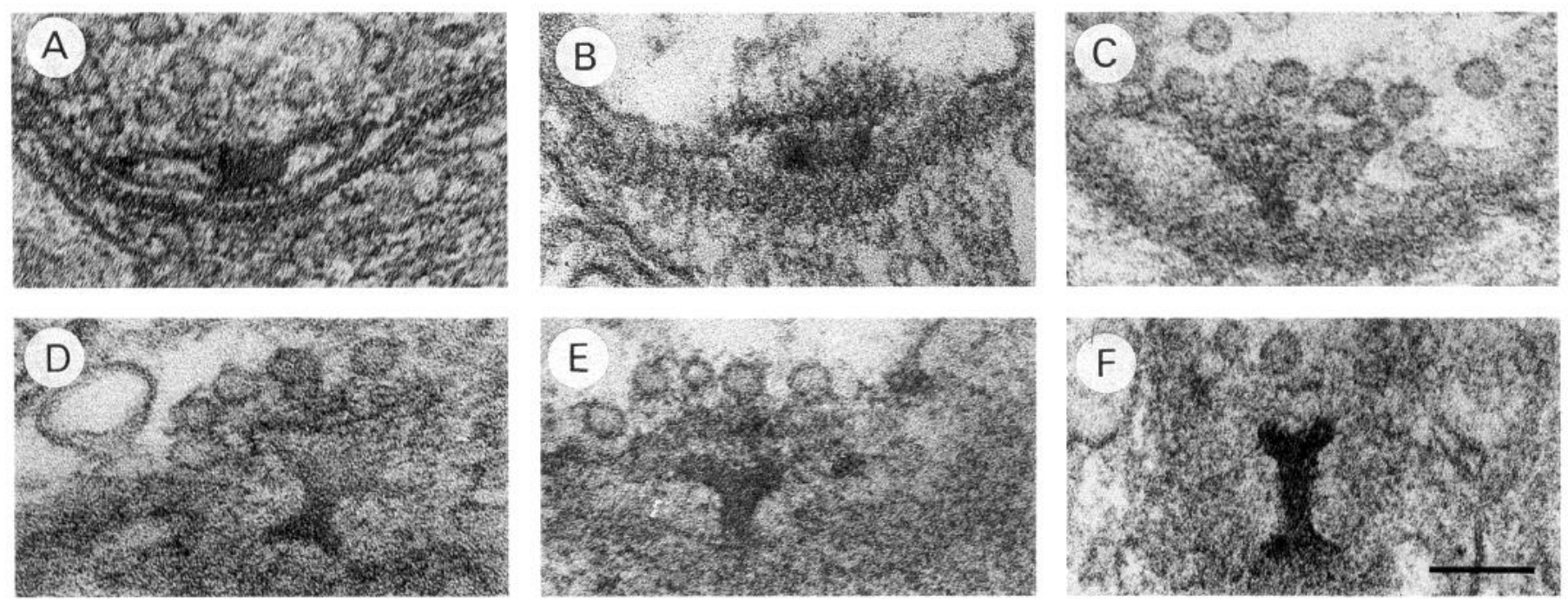

Figure 2. Examples of presynaptic dense body observed with different planes of sectioning. $A$, Dense body sectioned perpendicular to active site plasma membrane. $B$ and $C$, Tangential sections through dense body plate. $D-F$, Tangential sections through dense body base. Scale bar, $100 \mathrm{~nm}$ $(135,000 \times)$ for $A-F$.

For the experiment, the fly was mounted ventral side up in Tackiwax over an opening in a plastic tube and covered with saline. The thoracic and abdominal spiracles were left exposed to the air in the tube, allowing the fly to breathe. The preepisternum was dissected away to expose the coxal muscle. Glass capillary microelectrodes filled with $3 \mathrm{M} \mathrm{KCl}$ and having resistances of about $100 \mathrm{M} \Omega$ were used for the intracellular recordings. In some experiments the coxal nerve was cut to prevent efferent nerve impulses from the ganglion, while in other experiments the nerve was left intact. The intact preparation was in some cases stimulated by passing current through an electrode inserted into the thoracic ganglion. The experiments were all performed at room temperature $\left(24-26^{\circ} \mathrm{C}\right)$.

The normal saline solution (Bodenstein) consisted of $128 \mathrm{~mm} \mathrm{NaCl}$, $4.7 \mathrm{~mm} \mathrm{KCl}, 1.8 \mathrm{~mm} \mathrm{CaCl}_{2}$, and $5 \mathrm{~mm}$ Tris-aminomethane $\mathrm{HCl}(\mathrm{pH}$ 7.4). In some experiments, salines with elevated $\mathrm{Ca}^{2+}$ concentrations $(3.6,9.0,18 \mathrm{~mm})$ were used. These were prepared by increasing the $\mathrm{CaCl}_{2}$ and reducing the $\mathrm{NaCl}$ by an equiosmolar amount. In some experiments, a $0.1 \mathrm{~mm}$ solution of veratridine in normal saline was used. The body cavity was perfused with the experimental solutions through a capillary inserted into the anterioventral prothorax.

After the recording, the preparation was instantly perfused with fixative ( $2 \%$ paraformaldehyde, $2 \%$ glutaraldehyde in $0.1 \mathrm{~m}$ phosphate buffer, $\mathrm{pH}$ 7.4) for $30 \mathrm{~min}$. Since the naked terminals are located on the surface of the muscle fibers, fixation must occur very quickly, presumably in the order of milliseconds. Then, the coxal muscle was dissected out of the body and placed in $4 \%$ glutaraldehyde for $2 \mathrm{hr}$. The

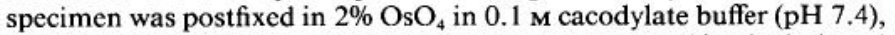
block-stained in $1 \%$ aqueous uranyl acetate, dehydrated in alcohol, and embedded in Spurr. Thin sections were double-stained with uranyl acetate and Millonig's lead hydroxide and observed on a Philips CM-10 electron microscope and photographed. In other experiments, specimens were exposed to normal and high-Ca ${ }^{2+}$ salines, and fixed without intracellular recording. In this case, fixative was poured directly on the naked terminals. In these specimens, the coxal nerve was cut.

The amplitudes of the miniature excitatory junction potentials (mejps), which were recorded on a photographic film directly from the oscilloscope, was measured using a micrometer attached to a stereomicroscope. For the distribution analysis, recordings that exhibited a stable mejp frequency that was low enough to distinguish between individual events were selected. In scoring mejps, those that had notches on their rising phases were scored as separate events.

\section{Results}

In Figure 1, a serially sectioned synapse of the coxal motor neuron is shown. The nerve was cut in this preparation to prevent activity from the ganglion. Attention should be directed to the two active sites (arrows) at this terminal. As can be seen, the pre- and postsynaptic membranes become closely apposed only at these sites. The active site region is defined postsynaptically by the postsynaptic membrane, which is thicker and more electron dense than the surrounding membrane. Extending into the synaptic cleft from the postsynaptic membrane is an electron-dense substance that in certain planes of sectioning appears as a brush-like array with a certain periodicity. Presynaptically, the active site is characterized by possessing a dense body. In individual sections, the dense body appears as a rectangular (inset in $C$ ) or round (inset in $D$ ) electron-dense base that is capped by a filamentous plate-like structure that attaches to the plasma membrane on either side of the dense body base by brush-like arrays of electron-dense material (inset in $D$ ).

In Figure 2, the dense body is shown in more detail. When the plane of sectioning is perpendicular to the active site plasma membrane, the typical image is that shown in Figure $2 A$. This sectioning plane reveals that the rectangular base of the dense body is actually slightly separated from the plasma membrane, and connected to it by three or four electron-dense strands. In Figure $2 \mathrm{~B}$, the plane of sectioning through the dense body is more tangential to the plasma membrane, revealing the filamentous nature of the dense body plate. In Figure $2 C$, another tangential section demonstrates the fanning out of the dense body plate toward synaptic vesicles. Tangential sections $D-F$ pass through the base of the dense body, revealing a dumbbellshaped structure that accounts for the round or rectangular shapes seen in perpendicular sections.

In Figure 1, the positioning of the synaptic vesicles relative to the dense body in a resting coxal synapse is demonstrated. As can be seen, the vesicles are located above and to the sides of the dense body, as well as more interiorly in the cytoplasm. No particular orderly positioning of vesicles relative to the dense body was seen in 340 resting coxal synapses observed. While vesicles were sometimes observed within a few nanometers of the plasma membrane on either side of the dense body at the active site, they were almost never observed under the dense body plate, close to the membrane adjacent to the base of the dense body. Only in 1 out of 340 synapses was a vesicle observed 
Figure 3. Example of typical vesicle positioning relative to the dense body $(A)$, and "docked" vesicle positioning (arrowheads) under dense body plate $(B)$ in resting coxal synapses. Note that the vesicle closest to dense body base appears to be attached to the plasma membrane by an electron-dense substance. Scale bars, $100 \mathrm{~nm}[112,500 \times$ $(A), 153,900 \times(B)]$.
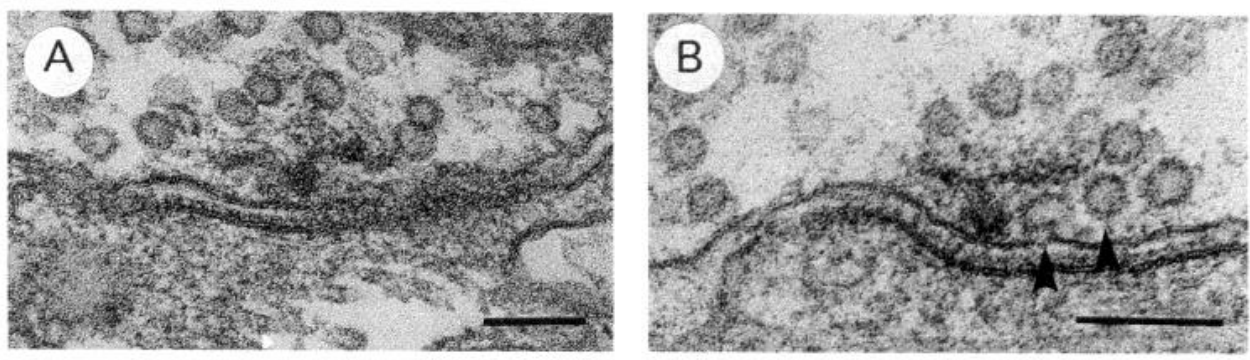

under the dense body plate (Fig. 3 ). Thus, the dense body plate actually appeared to present an obstacle to the vesicles touching the plasma membrane beneath it.

The effect of nervous activity on the positioning of the vesicles relative to the active site was next observed. Activity was induced either by electrically stimulating the nerve or by the ap- plication of veratridine. In Figure 4, $A-C$, typical synapses fixed during stimulation at $20 \mathrm{~Hz}$ are shown. Electrical stimulation of the synapse caused no detectable difference in the positioning of the vesicles relative to the dense body or active site. Often vacuoles were observed in these synapses, which appeared similar to those involved in the vesicle recycling process (Koenig
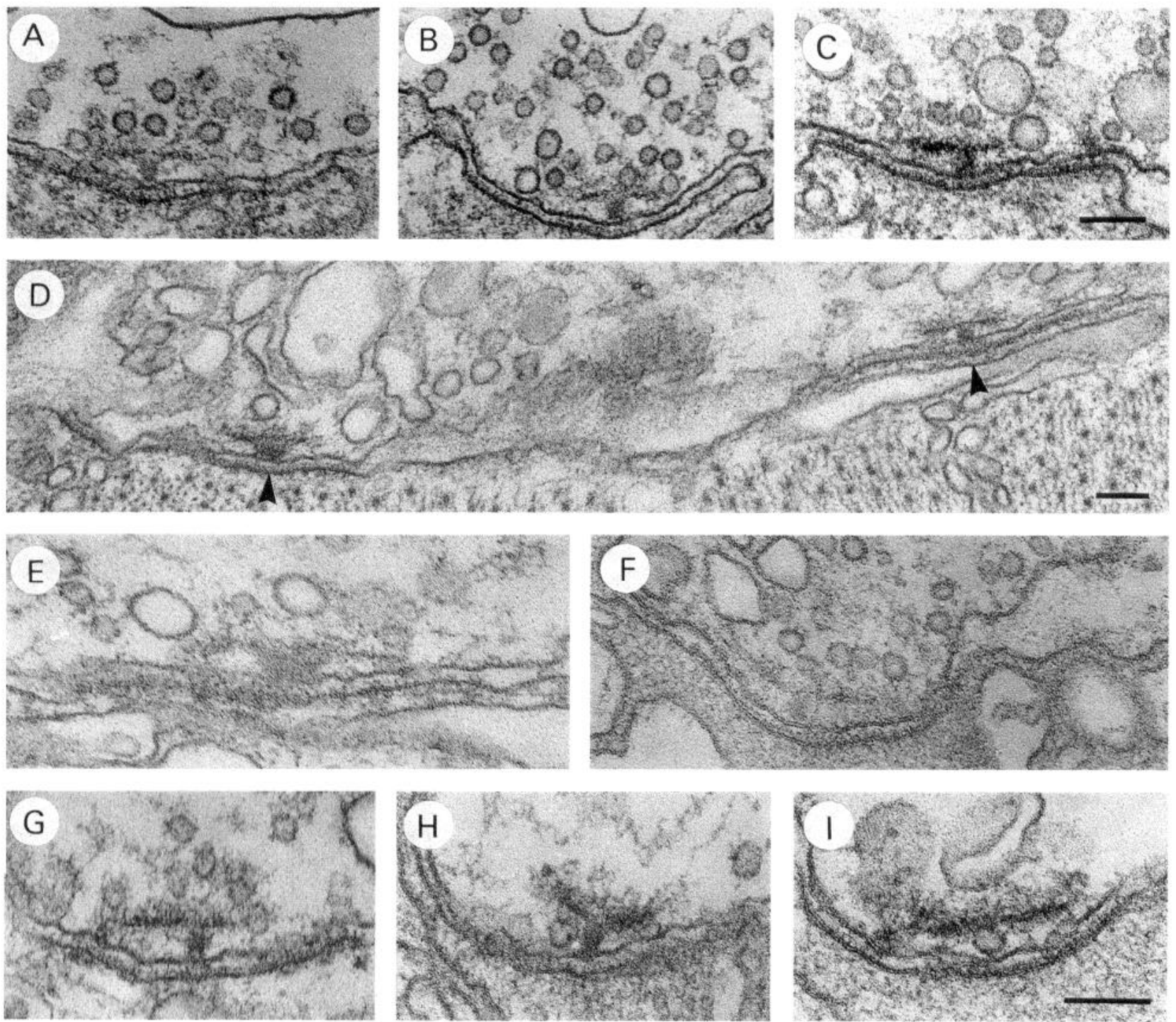

Figure 4. Examples of coxal active sites fixed during stimulation. $A-C$, Fixed while stimulating at $20 \mathrm{~Hz}$. Note the presence of vacuoles in $C$, which may represent a stage in the vesicle recycling process. $D-I$, Exposed to $0.1 \mathrm{~mm}$ veratridine for $1 \mathrm{~min}$. In $D$, two active sites (arrowheads) are completely depleted of synaptic vesicles. Note the cisternae, which are probably related to the vesicle recycling process. In $E-G$, partially depleted active sites with a few remaining synaptic vesicles are shown. Note that the vesicles are located above and to the sides of dense body plate. In $H$ and $I$, a single vesicle is located under the dense body plate. Scale bars: $C, 100 \mathrm{~nm}(90,000 \times)$ for $A-C ; D, 100 \mathrm{~nm}(75,000 \times) ; I, 100 \mathrm{~nm}(112,000 \times)$ for $E-I$. 

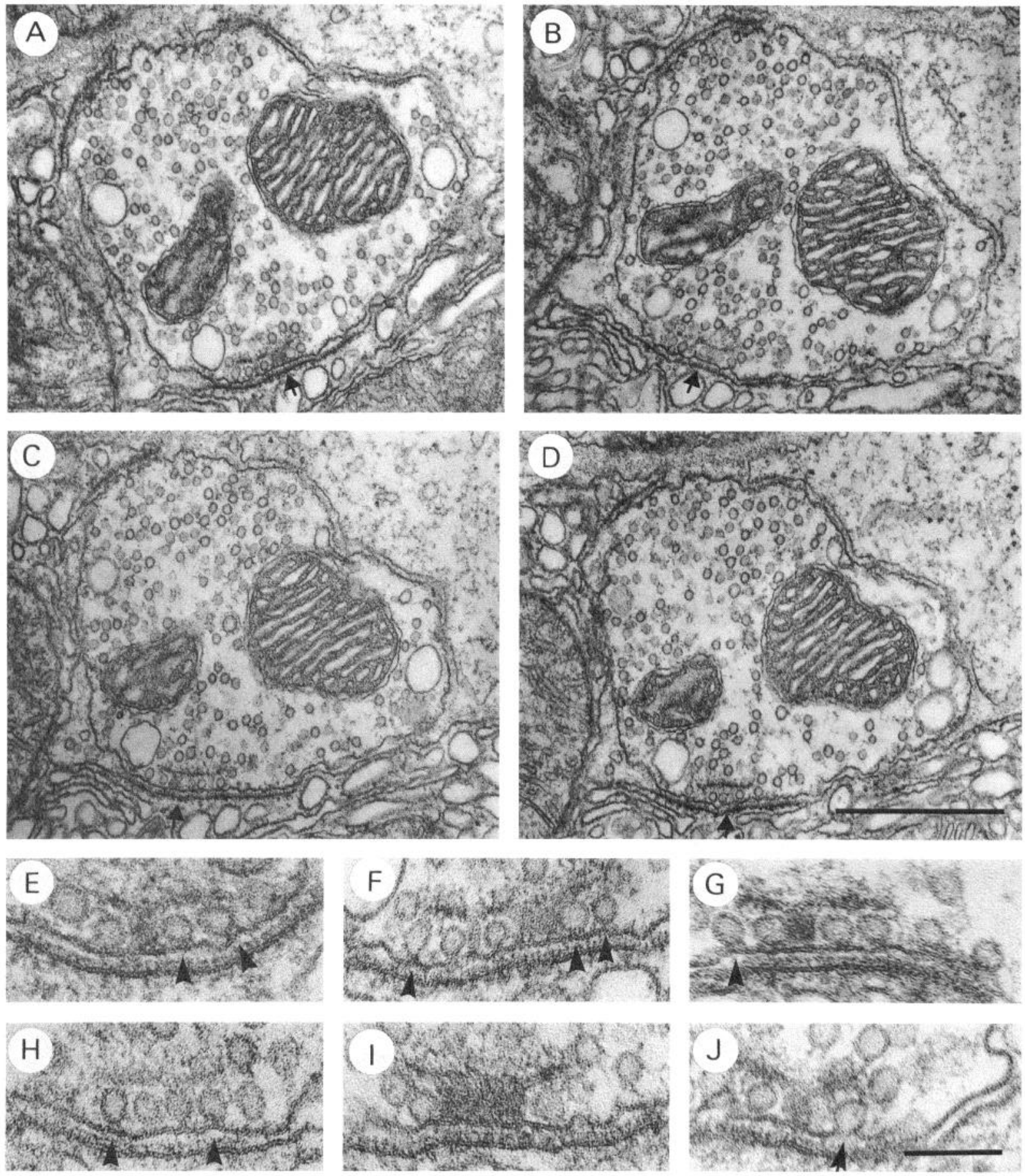

Figure 5. Example of coxal active sites after exposure to elevated $\mathrm{Ca}^{2+} . A-D$, Serial sections through a coxal synapse that had been exposed to $18 \mathrm{~mm} \mathrm{Ca}^{2+}$ saline for $10 \mathrm{~min}$. Note that several vesicles are located under the dense body plate at the active site in each section (arrows). E-J, Examples at higher magnification of typical active sites after exposure to $18 \mathrm{~mm} \mathrm{Ca}^{2+}$ for 10 min. Note that many of the vesicles appear to be attached to the presynaptic membrane by an electron-dense substance (arrowheads). In $J$, a possible exocytotic image is seen (arrow). Scale bars: $D, 0.5 \mu \mathrm{m}(54,000 \times)$ for $A-D ; J, 100 \mathrm{~nm}(157,000 \times)$ for $E-J$.

and Ikeda, 1989). After 1 min exposure to saline with $0.1 \mathrm{~mm}$ veratridine (Fig. $4 D-I$ ), partial or complete vesicle depletion was observed. In some cases, this resulted in a loss of vesicles from the interior of the synapse, with the few remaining vesicles positioned near the active sites. However, these remaining vesicles were almost always located above or to the sides of the dense body plate (Fig. $4 E-G$ ), as they were in the unstimulated synapse. In 2 instances out of 175 observed synapses, however, a vesicle was observed under the dense body plate (Fig. $4 H, I$ ). $\mathrm{No} \Omega$-shaped images suggesting exocytosis were seen under these conditions of stimulation.

In resting synapses that had been exposed to $18 \mathrm{mM} \mathrm{Ca}^{2+}$ saline for $10 \mathrm{~min}$ (nerve cut), a dramatic difference in the positioning of the vesicles relative to the presynaptic dense body 
Table 1. The effect of $\left[\mathrm{Ca}^{2+}\right]_{o}$ on the number of docked vesicles at the active site

\begin{tabular}{|c|c|c|}
\hline & $\begin{array}{l}\text { Sites with } \\
\text { docked vesicles }\end{array}$ & $\begin{array}{l}\text { Average } \\
\text { number of } \\
\text { docked } \\
\text { vesicles/site }\end{array}$ \\
\hline \multicolumn{3}{|c|}{$1.8 \mathrm{mM} \mathrm{Ca}^{2+}$ (Bodenstein) } \\
\hline$n=340(10$ flies $)$ & $0.3 \%$ & - \\
\hline \multicolumn{3}{|l|}{$3.6 \mathrm{mM} \mathrm{Ca}^{2+}(10 \mathrm{~min})$} \\
\hline$n=82$ (3 flies) & $56 \%$ & 1.4 \\
\hline \multicolumn{3}{|l|}{$18 \mathrm{~mm} \mathrm{Ca}^{2+}(10 \mathrm{~min})$} \\
\hline$n=120$ (5 flies) & $79 \%$ & 2.0 \\
\hline
\end{tabular}

A single thin section was made through each synapse. Docked vesicles are defined as those located under the dense body plate adjacent to the base of the dense body.

was observed. Now, many sites possessed vesicles under the dense body plate adjacent to the base of the dense body. This is demonstrated in the serially sectioned synapse shown in Figure $5, A-D$. In each section through this synapse, it can be seen that the active site possesses vesicles under the dense body plate. In Figure 5, $E-J$, examples of active sites from synapses exposed to $18 \mathrm{mM} \mathrm{Ca}^{2+}$ saline are shown with higher magnification. In these examples it can be seen that several vesicles are now located under the dense body plate adjacent to the dense body base. Also, it can be seen that, in most cases, the synaptic vesicle membrane is not in direct contact with the plasma membrane. Instead, many of the vesicles located under the plate appear to be attached to the presynaptic plasma membrane by an electrondense material (arrowheads). In some planes of sectioning, this material appears as two or more strands. For the remainder of this article, vesicles under the dense body plate will be referred to as "docked." Another difference in the $\mathrm{Ca}^{2+}$-exposed active sites was that occasionally exocytosis-like images were seen (Fig. $5 J)$. These $\Omega$-shaped images were observed only on the prcsynaptic membrane at the active site, and only under the dense body plate where the vesicles were also observed.

The percentages of active sites with vesicles under the plate in normal saline and elevated $\mathrm{Ca}^{2+}$ salines are shown in Table 1. It should be kept in mind that serial sectioning of every site was not done. Therefore, the values in Table 1 were arrived at by a single section through each site. Since each site spans a distance of about four to six thin sections (280 nm) (Figs. 1, 5), a site with no docked vesicles in one section may exhibit vesicles in the next section. Thus, these data demonstrate the increase in sites with vesicles in elevated $\left[\mathrm{Ca}^{2+}\right]_{o}$, but do not represent the actual percentage of sites with docked vesicles. From Table 1 it can be seen that as the $\left[\mathrm{Ca}^{2+}\right]_{0}$ is increased, the percentage of sites with docked vesicles increases dramatically. Also, the average number of docked vesicles per site increases. Again, it must be kept in mind that these averages are per single section through an active site. The actual number of docked vesicles per site is much larger than this, as can be seen in the serial sections shown in Figure $5, A-D$, which demonstrates that a single site may have 10 or more docked vesicles. The data of Table 1 demonstrate that the number of docked vesicles per site increases with increasing $\left[\mathrm{Ca}^{2+}\right]_{0}$.

\section{Electrophysiological observations}

The effect of incrcasing $\left[\mathrm{Ca}^{2+}\right]_{o}$ on spontaneous release at the coxal synapse was observed physiologically by recording intracellularly from coxal muscle fibers. One effect that was observed was an increase in the frequency of mejps in elevated $\mathrm{Ca}^{2+}$ saline over the initial frequency in normal saline $\left(1.8 \mathrm{mM} \mathrm{Ca}^{2+}\right)$. A typical example of a continuous intracellular recording from a coxal muscle fiber, first in normal saline and then in $9 \mathrm{~mm} \mathrm{Ca}^{2+}$ saline, is shown in Figure 6. A few mejps with amplitudes of about $0.5 \mathrm{mV}$ are seen in normal saline (Fig. 6A). An example of the spontaneous release from the same fiber after exposure to $9 \mathrm{mM} \mathrm{Ca}^{2+}$ for five $\mathrm{min}$ is shown in Figure $6 \mathrm{~B}$. As can be seen, the frequency of spontaneous release has increased. In Figure $6 C$, the frequency of spontaneous release after $10 \mathrm{~min}$ in $9 \mathrm{~mm} \mathrm{Ca}^{2+}$ is shown. The frequency eventually becomes so high that individual events cannot be distinguished.

Another effect that occurred after exposure to elevated $\mathrm{Ca}^{2+}$ saline was that there was an increase in the amplitude of individual mejps, as well as an increase in the release of mejps in clusters. This can be seen in Figure $6 B$, which demonstrates mejp clusters and doublets (arrowheads), and almost-synchronized and synchronized (multiquantal) mejps (arrows). The shift in mejp amplitude is demonstrated further in Figure 7, which
Figure 6. Example of a continuous intracellular recording from a coxal muscle fiber exposed first to normal saline $(A)$, and then to $9 \mathrm{mM} \mathrm{Ca}^{2+}$ saline for $5 \mathrm{~min}(B)$ and $10 \mathrm{~min}(C)$. Note the increase in frequency and amplitude of the mejps in elevated $\mathrm{Ca}^{2+}$ saline. Note the multiquantal mejps (arrows) and mejp clusters (arrowheads). Calibration: $5 \mathrm{mV}, 100 \mathrm{msec}$.
A
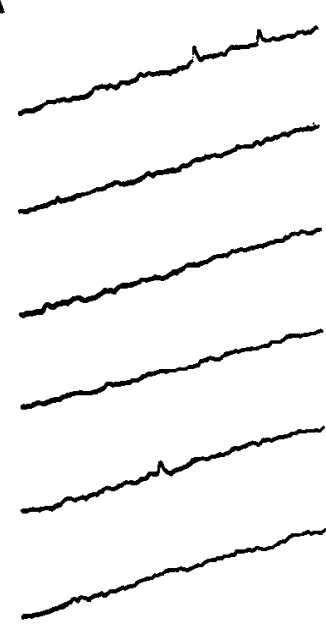

B

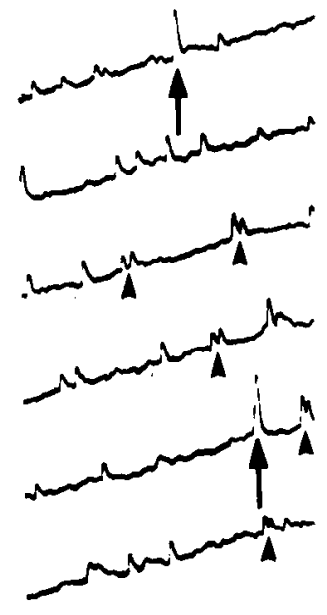

C
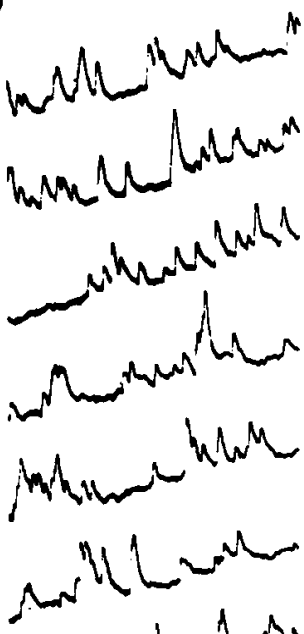

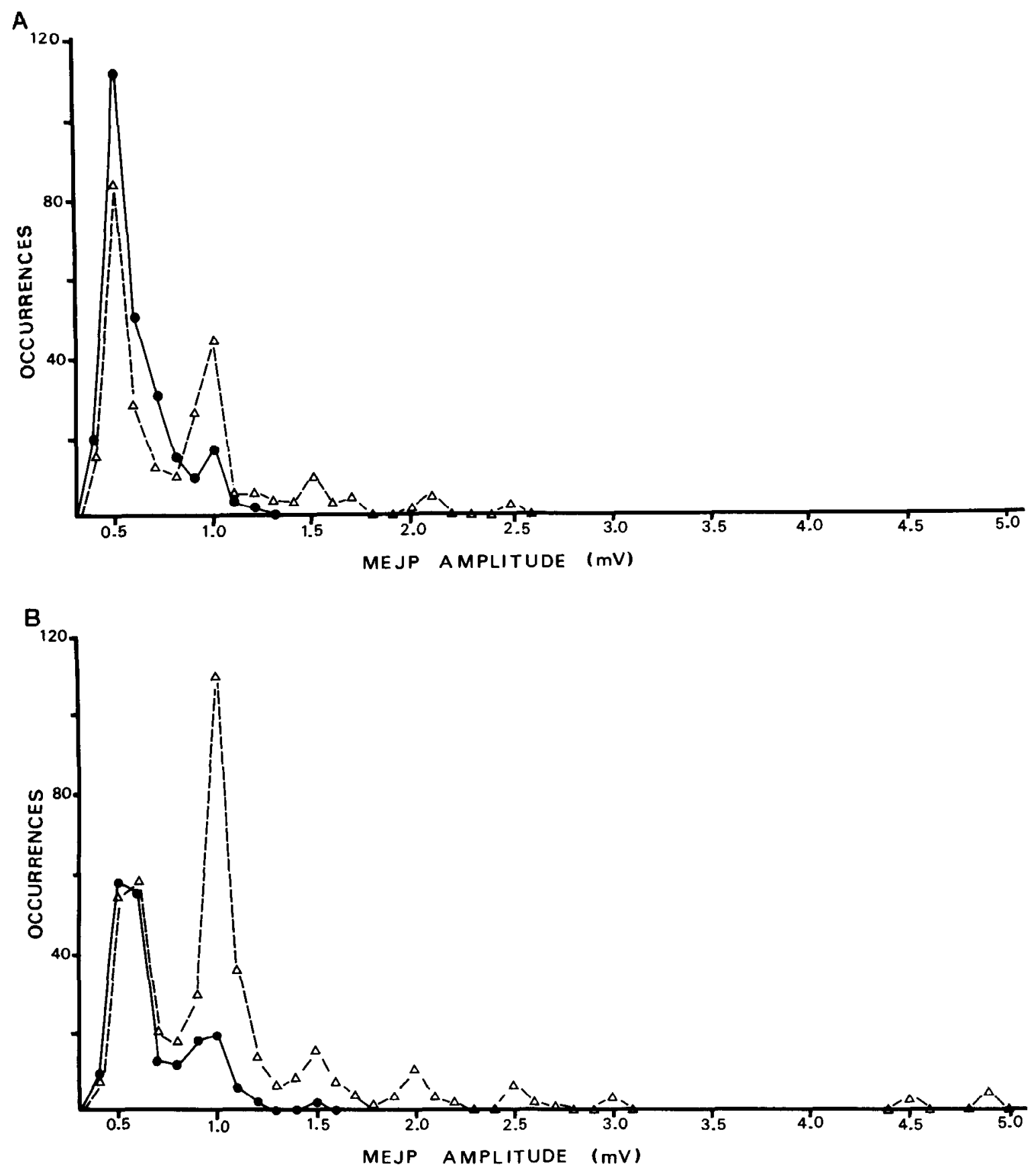

Figure 7. Two examples of mejp amplitude distributions from continuous intracellular recordings of coxal muscle fibers that were first exposed to normal saline $\left(\Theta\right.$, solid line) and then to elevated $\mathrm{Ca}^{2+}$ saline $\left(\triangle\right.$, broken line). $A, 3.6 \mathrm{~mm} \mathrm{Ca}{ }^{2+}$ for five min; $B, 18 \mathrm{~mm} \mathrm{Ca}{ }^{2+}$ for five min. Note shift to larger amplitudes in elevated $\mathrm{Ca}^{2+}$.

shows two typical examples of amplitude distributions taken from continuous intracellular recordings, first in normal saline (solid line), and then after exposure to elevated $\mathrm{Ca}^{2+}$ saline (broken line). In Figure $7 A$, the effect of exposure to $3.6 \mathrm{~mm}$ $\mathrm{Ca}^{2+}$ saline for five min is shown. As can be seen, the number of larger amplitude mejps is increased relative to that in normal saline. In Figure $7 B$, the effect of exposure to $18 \mathrm{mM} \mathrm{Ca}^{2+}$ for five $\min$ is shown. A greater increase in the number of largeramplitude mejps is now seen.

The amplitude distributions in elevated $\mathrm{Ca}^{2+}$ are multimodal, with peaks at roughly integral multiples of the smallest peak. This suggests that the larger events are multiquantal, that is, composed of synchronously occurring unitary events. The percentage of multiquantal mejps relative to unitary mejps varied from specimen to specimen. In normal saline, most of the mejps had amplitudes of about $0.5 \mathrm{mV}$, although some appeared multiquantal, having amplitudes of about $1 \mathrm{mV}$ or larger (Fig. $7 A, B$, solid lines). With exposure to high- $\mathrm{Ca}^{2+}$ saline, many more mejps were multiquantal, having amplitudes of about $1 \mathrm{mV}$ or larger (up to $5 \mathrm{mV}$ ) (Fig. $7 A, B$, broken lines). This increase in synchronously occurring unitary events was too frequent to be the result of randomly overlapping events. This is shown in Table 2 , which demonstrates that the occurrence of mejps in elevated $\left[\mathrm{Ca}^{2+}\right]_{o}$ does not fit a Poisson distribution, whether the large 
Table 2. Fit of mejp occurrences to a Poisson distribution with exposure to high-Ca ${ }^{2+}$ saline

\begin{tabular}{ccc} 
mejp in & $N_{x}$ & \\
\cline { 2 - 3 } sample $(x)$ & Observed & Expected \\
\hline With synchronized mejps scored as single events & \\
0 & 1064 & 1007 \\
1 & 474 & 575 \\
2 & 184 & 164 \\
3 & 50 & 31 \\
4 & 9 & 4 \\
With synchronized mejps scored as multiple events & \\
0 & 1064 & 463 \\
1 & 59 & 623 \\
2 & 210 & 420 \\
3 & 186 & 189 \\
4 & 118 & 64 \\
5 & 63 & 17 \\
6 & 34 & 4 \\
7 & 17 & 1 \\
8 & 14 & - \\
9 & 11 & - \\
10 & 5 & -
\end{tabular}

Data are fit of mejp occurrence to a Poisson distribution after exposure to $9 \mathrm{~mm}$ $\mathrm{Ca}^{2+}$ saline for $10 \mathrm{~min}$ in the coxal muscle. The expected values were derived from the equation $N_{x}=N_{\tau} e^{-\alpha} \alpha^{\gamma} / x !$, where $N_{T}$ is the total number of samples (10 msec bins), $N_{x}$ is the number of bins containing $x$ number of mejps, and $\alpha$ is the mean number of mejps per bin, calculated by dividing the total number of mejps by the total number of bins (1781). A, mejps with unnotched rising phases scored as single events regardless of amplitude. Total number of mejps $=1016, \alpha=0.57$. $B$, Same data as $A$, but with the larger than unitary size mejps with unnotched rising phases scored as multiple events, depending on amplitude. One mejp $=0.3$ $0.7 \mathrm{mV}$, two synchronized mejps $=0.8-1.4 \mathrm{mV}$, three synchronized mejps $=1.5$ $1.9 \mathrm{mV}$, etc., based on amplitude histogram for this recording. The total number of mejps increased to 2408 and $\alpha=1.35$.

mejps were scored as single events $(A)$ or multiquantal events $(B)$. The measure of agreement before the experimental and predicted distributions was determined using the $\chi^{2}$ test, and a probability of $<0.001$ was obtained. Thus, the distribution of mejps in $9 \mathrm{mM} \mathrm{Ca}^{2+}$ indicates that there is an increased probability that a particular mejp will fire synchronously with or soon after another mejp. The mejp amplitude distribution for the specimen used in Table 2 is shown in Figure 8 . The multimodal nature of this amplitude distribution is quite apparent.

\section{Discussion}

The data show that increasing $\left[\mathrm{Ca}^{2+}\right]_{0}$ influences synaptic vesicles to translocate to a readily releasable position under the dense body plate at the active site. Many of these vesicles appeared to be attached to the plasma membrane by electrondense strands, suggesting they may represent a population of "docked" vesicles in a readily releasable state. Since this effect was observed in the absence of any nervous activity, it must be assumed that even though the resting permeability to $\mathrm{Ca}^{2+}$ is low, increasing the $\mathrm{Ca}^{2+}$ gradient across the terminal membrane by increasing $\left[\mathrm{Ca}^{2+}\right]_{o}$ causes an increase in $\left[\mathrm{Ca}^{2+}\right]_{i}$. Although the magnitude of the concentration change inside the terminal is not known, the data do demonstrate that $\left[\mathrm{Ca}^{2+}\right]_{i}$ influences the positioning of vesicles relative to the active site, and furthermore, they demonstrate that the greater the increase in $\left[\mathrm{Ca}^{2+}\right]_{i}$, the greater the number of sites possessing docked vesicles, and the greater the average number of docked vesicles per site.
As mentioned in Results, the data presented here do not indicate precisely the percentage of sites with docked vesicles, since serial sectioning was done on only a limited number of sites. For example, the data show that in 340 sections through different resting synapses in normal saline, only one demonstrated a vesicle docked under the dense body plate. However, if serial sectioning had been done on each of these 340 synapses, it is probable that some more would have been found. These data simply demonstrate that the percentage of sites with docked vesicles must be quite low. On the other hand, $79 \%$ of the observed sections through active sites exposed to $18 \mathrm{mM} \mathrm{Ca}^{2+}$ for $10 \mathrm{~min}$ showed at least one docked vesicle. However, the actual percentage of active sites possessing docked vesicles is probably higher than this.

The mode of action of $\mathrm{Ca}^{2+}$ to influence the vesicles to translocate under the dense body plate cannot be determined by these results. However, recent biochemical and ultrastructural observations may give insight into this question. In recent years, a number of $\mathrm{Ca}^{2+}$-binding proteins that are associated with synaptic vesicle membrane have been isolated and characterized. These include F-actin, fodrin, and the synapsins. Of particular interest is synapsin I, which has been shown to bind to small clear synaptic vesicles, F-actin, and fodrin in vitro (Baines and Bennett, 1985; Schiebler et al., 1986). Phosphorylation of synapsin I by $\mathrm{Ca}^{2+} /$ calmodulin (CM) kinase II reduces the affinity of binding to vesicles (Schiebler et al., 1986) as well as to F-actin (Bahler and Greengard, 1987). Ultrastructural evidence using quick-freeze etching and rotary replication techniques demonstrate synapsin-like molecules linking synaptic vesicles to a network of actin-like filaments associated with the active zone. Also, fodrin-like strands have been shown to radiate from the active zone presynaptic membrane to both the actin-like and synapsin-like filaments (Landis et al., 1988; Hirokawa et al., 1989). Thus, as mentioned in the introductory remarks, it has been proposed that phosphorylation of synapsin I may release vesicles from the cytonet, increasing vesicle availability for transmission. Indeed, it has been shown that injection of the dephosphorylated form of synapsin into the presynaptic terminal of the squid giant axon inhibited transmission, while injection of the phosphorylated form did not. Furthermore, injection of $\mathrm{Ca}^{2+} / \mathrm{CM}$ kinase II facilitated transmission (Llinas et al., 1985).

The data presented here are consistent with the possibility that $\mathrm{Ca}^{2+}$ may be involved in releasing vesicles from the cytonet. Thus, the resting $\left[\mathrm{Ca}^{2+}\right]_{i}$ may regulate the percentage of sites that possess readily releasable vesicles. Exactly how the freed vesicles arrive under the dense body plate, which appears to present a physical barrier to them, is not clear, however. From the active site architecture at this synapse, it seems probable that these vesicles must be drawn to the presynaptic membrane at the active site in some way, either mechanically or by electrical attraction, rather than arriving there from random movement. The role of $\mathrm{Ca}^{2+}$ in this process is not determined.

The data further demonstrate the effect of elevated $\left[\mathrm{Ca}^{2+}\right]_{o}$ on the spontaneous release of mejps. One effect was an increase in the frequency of spontaneous release. This effect has been reported previously at other synapses (Blioch et al., 1968; Hubbard et al., 1968; Bornstein, 1978). An increase in frequency might be expected if the number of sites possessing readily releasable vesicles were increased. Also, $\mathrm{Ca}^{2+}$ may act directly on the spontaneous release mechanism. A second effect was that the probability of synchronous and clustered release of unitary 


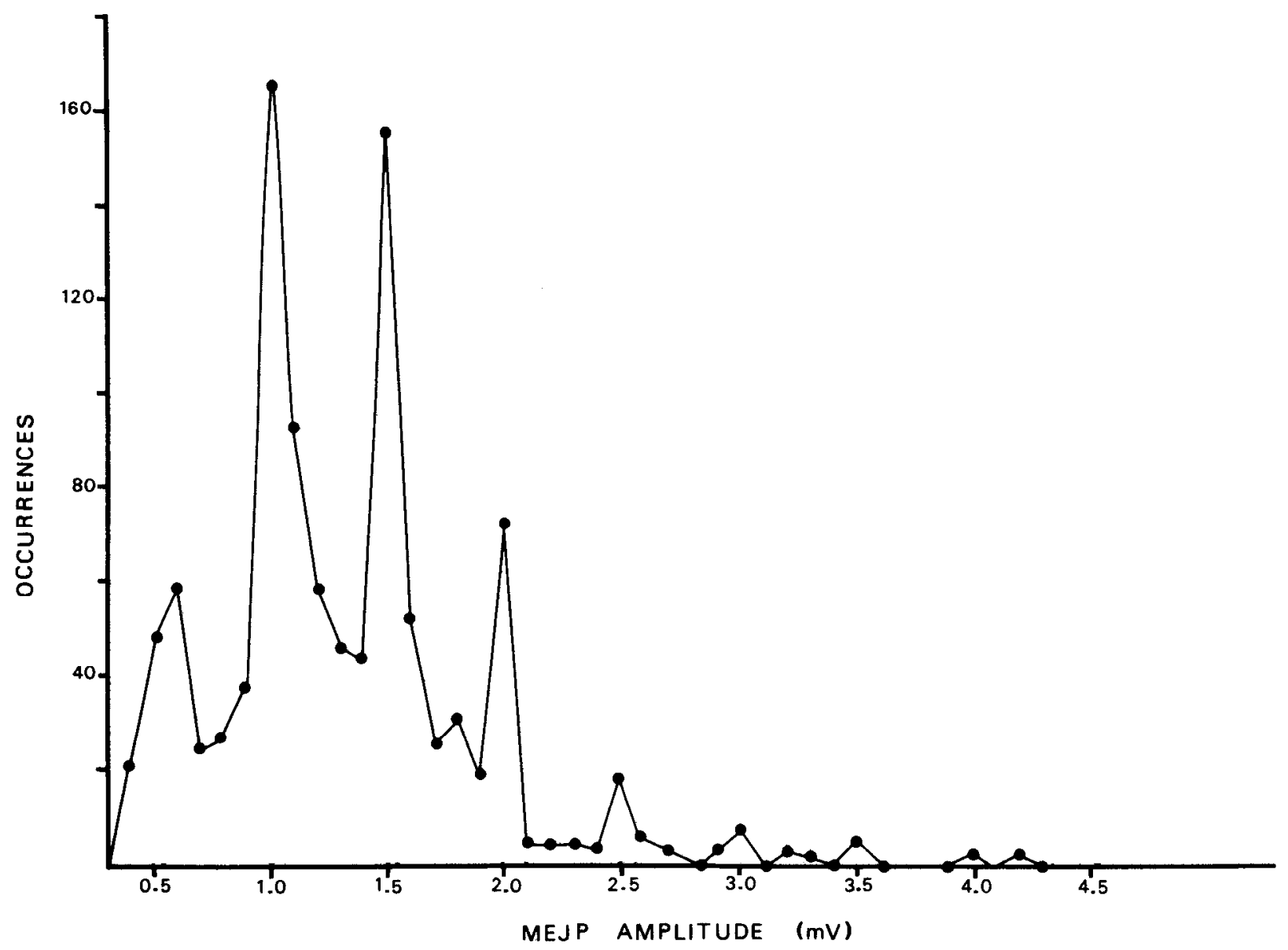

Figure 8. Amplitude distribution from a coxal muscle after exposure to $9 \mathrm{~mm} \mathrm{Ca}^{2+}$ saline for $10 \mathrm{~min}$. Note multimodal nature of distribution.

events increased. It has been shown previously with other preparations that increasing $\left[\mathrm{Ca}^{2+}\right]_{o}$ increases the probability of multiquantal and clustered spontaneous release (Rothshenker and Rahamimoff, 1970; Dennis et al., 1971; Bornstein, 1978). Focal extracellular recordings suggest that these multiquantal mejps or clusters of mejps occur at individual active sites rather than from an interaction between several different sites (Cohen et al., 1974; Rees, 1974). However, the mechanism responsible for this phenomenon was not known. The present data demonstrate an increase in the number of docked vesicles per active site with increased $\left[\mathrm{Ca}^{2+}\right]_{o}$. Thus, it is possible that a multiquantal mejp, or a cluster of almost synchronized mejps, may arise from a single active site at which more than one vesicle is in a readily releasable state. Possibly, a single site releases all of its readily releasable vesicles more or less synchronously in an all-or-none fashion.

One explanation for the above observations is as follows. The spontaneous release of a single active site can result in a unitary mejp, a multiquantal mejp, or a tight cluster of mejps, depending on how many vesicles are in a readily releasable state at that site. In normal saline, only a small percentage of sites possess one readily releasable vesicle, and rarely two or more. This would give mejp amplitude distributions such as seen in Figure 7 (solid lines). Thus, an all-or-none spontaneous release event at a given site would usually result in a unitary mejp. In elevated $\left[\mathrm{Ca}^{2+}\right]_{o}$, however, many sites may now possess more than one readily releasable vesicle, and therefore will produce a multi- quantal event, giving multimodal amplitude distributions as seen in Figure 7 (broken lines).

The fact that, under normal conditions, most sites appear not to possess any vesicles in a readily releasable position is not surprising, since many hundreds of sites exist on a single coxal fiber, and it would not be necessary for all of them to contribute a single quantum in response to a nerve impulse. A similar situation was shown with the neuromuscular junctions of the dorsal longitudinal flight muscle (DLM). A single fiber of this muscle is innervated by thousands of active sites. When partial vesicle depletion was induced using the shibire mutant at $29^{\circ} \mathrm{C}$, so that the excitatory junction potential (ejp) amplitude was reduced to $2 \mathrm{mV}$, it was observed morphologically that while the majority of the synapses had become totally depleted of vesicles, many others were only partially depleted, still possessing at least one vesicle and often more. Since each quantum produced a response of about $0.5 \mathrm{mV}$ in this muscle, this demonstrated that only about four quanta $(2 \mathrm{mV})$ were released per impulse, even though many hundreds of vesicles were present at the many synapses on this fiber. Apparently, only a very small percentage of the vesicles present at these synapses was in a readily releasable state (Koenig et al., 1989). At the time this work was done, a possible morphological correlate for the "readily relcasable" statc had not bcen identified. However, the present data suggest that one prerequisite may be positioning under the dense body plate.

The fact that exocytosis-like images were not observed in 
stimulated synapses, yet were occasionally observed in resting synapses exposed to elevated $\left[\mathrm{Ca}^{2+}\right]_{0}$ where the only release was spontaneous, is difficult to understand. It has been suggested that aldehyde fixation may cause slowing of the exocytosis process, so that the probability of catching such an event is increased (Heuser et al., 1974). In our preparation using 2\% paraformaldehyde- $2 \%$ glutaraldehyde, we found no suggestion of this effect, however. One explanation for observing exocytotic images at active sites exposed to elevated $\mathrm{Ca}^{2+}$ is that $\mathrm{Ca}^{2+}$ slows down exocytosis in some way, making it more probable that an event would be observed. However, there is no suggestion of such an effect physiologically. A more likely possibility is that the number of active sitcs with readily relcasable vesicles is incrcascd in elevated $\mathrm{Ca}^{2+}$, thus increasing spontaneous release, which increases the probability of release events occurring at the moment of fixation. If many different sites are observed, a release event may be encountered. One possible reason why it is so difficult to catch exocytotic events upon stimulation is that in neuromuscular junctions only a small fraction of the many active sites release for any given impulse. Thus, the chance of encountering by electron microscopic examination an active site that had responded to a particular stimulus might be low.

The data presented here suggest that the concentration of $\mathrm{Ca}^{2+}$ inside the terminal may regulate the number of sites that possess readily releasable vesicles. Under normal resting conditions in the coxal motor neuron, this number appears to be quite low. The influx of $\mathrm{Ca}^{2+}$ that would occur during nervous activity could transiently increase this number. However, preparations fixed during moderate stimulation did not reflect this. Only in preparations exposed to veratridine, which causes massive release, did an increase in "docked" vesicles possibly occur. Prob$\mathrm{ably}$, the $\mathrm{Ca}^{2+}$ that enters the terminal during an action potential is utilized for the release event itself, and not for translocation of vesicles to the active site. The latter may be primarily under the control of cytoplasmic stores of $\mathrm{Ca}^{2+}$.

\section{References}

Atlas D (1990) The role of calcium in neurotransmitter release: existing models and new approaches to evaluate possible mechanisms. Curr Top Cell Regul 31:129-159.

Augustine GJ, Charldon MP, Smith SJ (1987) Calcium action in synaptic transmitter release. Annu Rev Neurosci 11:458-464.

Bahler M, Greengard P (1987) Synapsin I bundles F-actin in a phosphorylation-dependent manner. Nature 326:704-707.

Baines AJ, Bennett V (1985) Synapsin I is a spectrin-binding protein immunologically related to erythrocyte protein 4.1. Nature 315:410413.
Berl S, Puszkin S, Nicklas WJ (1973) Actomyosin-like protein in brain. Science 179:441-446.

Blioch ZL, Glagoleva IM, Lieberman EA, Nenashev VA (1968) A study of the mechanism of quantal transmitter release at a chemical synapse. J Physiol (Lond) 199:11-35.

Bornstein JL (1978) Multiquantal release at synapses in guinea-pig hypogastric ganglia: evidence that release can occur in bursts. J Physiol (Lond) 282:375-398.

Cohen I, Kita H, Van der Kloot W (1974) The stochastic properties of spontaneous quantal release of transmitter at the frog neuromuscular junction. J Physiol (Lond) 236:341-361.

Curran MJ, Brodwick MS (1984) Exocytosis in permeabilized mast cells. Biophys J 45:170a.

del Castillo J, Katz B (1956) Biochemical aspects of neuro-muscular transmission. Prog Biophys Chem 6:122-170.

Dennis MJ, Harris AJ, Kuffler SW (1971) Synaptic transmission and its duplication by focally applied ACh in parasympathetic neurons in the heart of the frog. Proc R Soc Lond [Biol] 177:509-539.

Edwards C, Englert D, Lotshaw D, Ye HZ (1984) Light microscopic observations on the release of vesicles by isolated chromaffin cells. Cell Motil 4:297-303.

Heuser JE, Reese TS, Landis DMD (1974) Functional changes in frog neuromuscular junctions studied with freeze-fracture. J Neurocytol 3:109-131.

Hirokawa N, Sobue K, Kanda K, Harada A, Yorifuji H (1989) The cytoskeletal architecture of the presynaptic terminal and molecular structure of Synapsin I. J Cell Biol 108:111-126.

Hubbard JI, Jones SF, Landau EM (1968) On the mechanism by which calcium and magnesium affect the spontaneous release of transmitter from mammalian motor nerve terminals. J Physiol (Lond) 194:355380.

Katz B, Miledi R (1965) The effect of calcium on acetylcholine release from motor nerve terminals. Proc R Soc Lond [Biol] 161:496-503.

Koenig JH, Ikeda K (1989) The disappearance and reformation of synaptic vesicle membrane upon transmitter release observed under reversible blockage of membrane retrieval. J Neurosci 9:3844-3860.

Koenig JH, Kosaka T, Ikeda K (1989) The relationship between the number of synaptic vesicles and the amount of transmitter released. J Neurosci 9:1937-1942.

Landis DMD, Hall AK, Weinstein LA, Reese TS (1988) The organization of cytoplasm at the presynaptic active zone of a central nervous system synapse. Neuron 1:201-209.

Llinas R, McGuiness TL, Leonard CS, Sugimori M, Greengard P (1985) Intraterminal injection of synapsin I or calcium/calmodulin-dependent protein kinase II alters neurotransmitter release at the squid giant synapse. Proc Natl Acad Sci USA 82:3035-3039.

Rees D (1974) The spontaneous release of transmitter from insect nerve terminals as predicted by a negative binomial theorem. J Physiol (Lond) 236:129-142.

Rothshenker S, Rahamimoff R (1970) Neuromuscular synapse: stochastic properties of spontaneous release of transmitter. Science 10: 648-649.

Schiebler W, Jahn R, Doucet J-P, Rothlein J, Greengard P (1986) Characterization of synapsin I binding to small synaptic vesicles. J Biol Chem 261:8383-8390. 